\title{
Photostability of gas- and solid-phase biomolecules under astrophysical analog soft $\mathrm{X}$-rays field
}

\author{
S. Pilling ${ }^{1,2}$, D. P. P. Andrade ${ }^{1,2}$, R. T. Marinho ${ }^{3}$, E. M. do \\ Nascimento $^{3}$, H. M. Boechat-Roberty ${ }^{4}$, R. B. de Castilho ${ }^{4}$, G. G. B. \\ de Souza $^{4}$, L. H. Coutinho ${ }^{4}$, R. L. Cavasso-Filho ${ }^{5}$, A. F. Lago $^{5}$, and \\ A. N. de Brito ${ }^{6}$ \\ ${ }^{1}$ Pontifícia Universidade Católica do Rio de Janeiro (PUC-Rio), Rio de Janeiro, RJ, Brazil. \\ ${ }^{2}$ Universidade do Vale do Paraíba (UNIVAP/IP\&D), São José dos Campos, SP, Brazil. \\ ${ }^{3}$ Universidade Federal da Bahia (UFBA), Bahia, BA, Brazil. \\ ${ }^{4}$ Universidade Federal do Rio de Janeiro (UFRJ), Rio de Janeiro, RJ, Brazil. \\ ${ }^{5}$ Universidade Federal do ABC (UFABC), São Paulo, Brazil. \\ ${ }^{6}$ Laboratório Nacional de Luz Síncrotron (LNLS), Campinas, SP, Brazil.
}

\begin{abstract}
We present experimental studies on the interaction of soft X-rays on gas-phase and solid-phase amino acids and nucleobases in an attempt to verify if these molecules (supposed to be formed in molecular clouds/protostellar clouds) can survive long enough to be observed or even to be found in meteorites. Measurements have been undertaken employing $150 \mathrm{eV}$ photons under high vacuum conditions at the Brazilian Synchrotron Light Laboratory (LNLS). The produced ions from the gas-phase experiments (glycine, adenine and uracil) have been mass/charge analyzed by time-of-flight spectrometer. The analysis of solid phase samples (glycine, DL-proline, DL-valine, adenine and uracil) were performed by a Fourier transform infrared spectrometer coupled to the experimental chamber. Photodissociation cross sections and halflives were determined and extrapolated to astrophysical environments. The nucleobases photostability was up to two orders of magnitude higher than for the amino acids.
\end{abstract}

Keywords. astrochemistry, astrobiology, molecular processes, molecular data, methods: laboratory, ISM: clouds, X-rays: ISM

\section{Introduction}

The search for amino acids, nucleobases and related compounds in the interstellar medium/comets has been performed at least in the last 30 years. Recently, some traces (upper limits) of these molecules (e.g. glycine and pyrimidine) have been detected in molecular clouds (MC), protoplanetary disks (PPD) and in comets (Kuan et al. (2003); Elsila et al. (2009)). The search for these biomolecules in meteorites, on the contrary, has been revealed an amazing number, up to several parts per million (Kissel \& Krueger (1987)). This chemical dichotomy between the molecular inventories found in meteorites and interstellar medium and comets remains a big puzzle in astrochemistry field and in the investigation about the origin of life.

\section{Experimental methodology and results}

To verify if these biomolecules (supposed to be formed in MC or PPD) can survive long enough to soft X-ray exposition to be observed or even to be found in meteorites, we have 
used the Brazilian Synchrotron Light Laboratory (LNLS) facility, located at Campinas, Brazil. The measurements have been undertaken employing $150 \mathrm{eV}$ photons $\left(\sim 4 \times 10^{11}\right.$ photons $\mathrm{cm}^{-2} \mathrm{~s}^{-1}$ ) from the toroidal grating monocromator (TGM) beamline under high vacuum conditions. For the gas-phase experiments (glycine, adenine and uracil) the soft X-ray beam had intercepted perpendicularly the molecular beam. The produced ions were mass/charge analyzed by Time-of-Flight mass spectrometry (TOF-MS) employed in a photoelectron-photoion coincidence mode (Figure 1a). In the case of solid phase samples (glycine, DL-proline, DL-valine, adenine and uracil), in-situ analysis were performed by a Fourier transform Infrared spectrometry (FTIR) coupled to the experimental chamber (Figure 1b). Further experimental information is given elsewhere (Pilling et al. (2009))

The results show that the gaseous amino acids are virtually destroyed by soft Xrays while the nucleobases have a survivability of a few percents. The dissociation cross section of glycine in the gas phase is about 10 times lower than in the solid-phase. In the case of nucleobases both solid and gas-phase present similar dissociation cross section. Condensed amino acids can survive at least $\sim 7 \times 10^{5}$ and $\sim 7 \times 10^{8}$ years into dense $\mathrm{MC}$ and PPD, respectively.
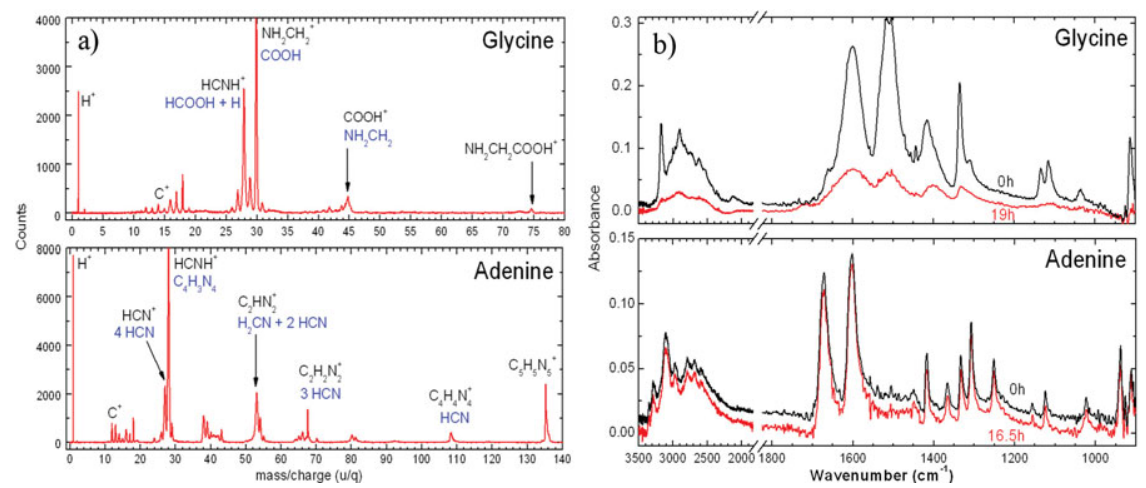

Figure 1. a) Mass spectra of the ionic species produced by the interaction of $150 \mathrm{eV}$ photons on two gaseous samples. b) Infrared spectra of two solid-phase samples before and after irradiation by $150 \mathrm{eV}$ photons. Details can be found elsewhere (Pilling et al. (2009))

\section{Conclusions}

Experimental study on the interaction of $150 \mathrm{eV}$ photons on gaseous and solid-phase biomolecules expected to be found interstellar dense clouds have been performed. The nucleobases photostability is up to two orders of magnitude higher than for the amino acids. The determined lifetime for these molecules is of the order or even grater than $\mathrm{MC}$ and PPD lifetime. This result corroborates the scenario in which during planetary formation (and after) these molecules, trapped into and onto dust grains, meteoroids and comets, could be delivered into the planets/moons possibly allowing pre-biotic chemistry in such environments where water was also found in liquid state.

\section{References}

Elsila, J., et al. 2009, Met. ES Planetary Science, In press

Kissel, J. \& Krueger, F. R. 1987, Nature, 326, 755

Kuan, Y.-J., Charnley, S. B., Huang, H.-C., Tseng, W.-L., \& Kisiel, Z. 2003, ApJ, 593, 848

Pilling, S., Andrade, D. P. P., Marinho R. T., et al. 2009, A\&A, to be submited 